\title{
El azar como espacio positivo de indeterminación en la asimilación tomista de la física de Aristóteles
}

\author{
Ana Maria Minecan \\ CRNS - Institut de Recherche et Histoire des Textes, Francia
}

Resumen: El presente artículo se centra en el análisis de la asimilación por parte de Tomás de Aquino de la teoría aristotélica del azar. Se muestran los puntos en los cuales el Aquinate aceptó sin restricciones la visión de Aristóteles, así como aquellas cuestiones en las cuales el dominico se separó de la posición aristotélica ofreciendo una nueva interpretación del papel del azar dentro del cosmos creado. La confluencia crítica de estos dos grandes sistemas de pensamiento -el aristotelismo y el cristianismo tardío medieval- dieron paso a nuevas formas de comprender la naturaleza al ampliar el ámbito de estudio de la física desde lo absolutamente determinado hacia lo anómalo e irregular.

Palabras clave: filosofia de la naturaleza; fisica medieval; cosmologia; azar; determinismo

\begin{abstract}
Chance as a Positive Space of Indetermination in the Thomistic Assimilation of Aristotle's Physics". This article analyzes Thomas Aquinas's assimilation of the Aristotelian theory of chance. The analysis highlights Thomas Aquinas's unequivocal acceptance of Aristotelian philosophy, as well as the issues regarding which he abandoned the Aristotelian position by offering a new interpretation related to the role of chance in the context of the created cosmos. All in all, the confluence of these two great systems of thought-Aristotelian thought and late Medieval Christianity- provided new ways to understand nature, by expanding the scope of physics from the absolutely determined to the anomalous and irregular. Keywords: philosophy of nature; medieval physics; cosmology; chance; determinism
\end{abstract}




\section{El azar en la física de Aristóteles}

La naturaleza, tal y como se presenta en la Física de Aristóteles, se caracteriza por estar necesariamente determinada en sus procesos por un conjunto de principios inalterables (Física f. 63-79. II, 4-6) ${ }^{1}$. El carácter regular, cíclico y determinado, en el cual la concatenación causal orientada teleológicamente se efectúa "siempre o en la mayoría de los casos" (f. 82, 3-6. II, 5, 198b10-13), define la esencia misma de lo fisico. La propia arquitectura de lo natural, configurada por la interacción de cuatro causas, dos principios contrarios y una materia subyacente diversificada en los cuatro elementos simples, establece para cada entidad un conjunto de procesos determinados tanto en lo que respecta a la acción propia de cada cosa, como al conjunto de fenómenos que esta es capaz de padecer ${ }^{2}$. En este cosmos, legislado de forma universal y necesaria por las normas intrinsecas derivadas del binomio forma-materia, lo azaroso o accidental -entendido como aquello que se desarrolla de modo anómalo respecto a tales determinaciones y acerca de lo cual no es posible determinar de forma clara causas específicas- constituye un problema cuyo impacto el Estagirita constantemente intentó minimizar con el fin de salvaguardar el carácter ordenado de lo real y garantizar su alcance epistemológico por parte del ser humano.

No obstante, a pesar de las dificultades que a nivel ontológico supuso para la arquitectura aristotélica de lo natural la aceptación del azar, Aristóteles no rechazó su estudio, sino que admitió la presencia de un conjunto de procesos que se sustraen a la mecánica habitual del mundo físico. En opinión del Estagirita, la propia observación empírica (f. 66, 9-11. II, 4, 196b2-4) ${ }^{3}$ revela la existencia de un conjunto de fenómenos cuya explicación remite a la causación accidental (f. 71, 4-6. II, 5, 197a11-14)

\footnotetext{
1 Las fuentes primarias del artículo se apoyan en las traducciones latinas del siglo XIII de la obra física de Aristóteles, tal y como esta fue leída por Tomás de Aquino. Por ello, citaremos primero la versión latina seguida de la correspondencia de la misma en la edición Bekker.

2 Cunningham, H-P., Négation de la négation, Á propos de "hazard" et de "nécésité", Quebec: Lava, 1972, p. 21.

3 "Ad id enim et aliter inconveniens ese quod dictum est, adhuc inconveniens est dicere hec videntes in celo quidem nichil a causa fieri, in his autem que non sunt a fortuna multa contingere a fortuna; et merito etiam erat contrario fieri".

4 "Omne enim hec recte dicuntur, quoniam rationabiliter. Est quidem enim cum fit a fortuna".
} 
En el libro II de la Física, Aristóteles hizo uso de la noción de casualidad para referirse al ámbito del azar propio de los procesos relativos a los compuestos hilemórficos. Con este término, el filósofo griego pretendió designar las causas accidentales que intervienen cuando a una entidad dotada de finalidad le sobreviene algo que difiere de su fin propio $^{5}$. Para enfatizar este hecho, Aristóteles sostuvo que el término casualidad equivale a la expresión "en vano" empleada cuando no se logra aquello para lo cual se ha hecho algo. Así, algo es en vano cuando lo que está dispuesto por naturaleza para una cosa no cumple aquello para lo cual está naturalmente orientado (f. 76, 1-5. II,6, 197b22-27)6 .

Por lo tanto, las cosas que son producidas accidentalmente no tienen en sí el principio de su producción, al contrario de lo que ocurre con las cosas que son por naturaleza (f. 42, 3-6. II, 1, 192b21)7 , sino que unas lo tienen fuera, en otras cosas, y otras lo tienen en sí mismas pero no por sí mismas (f. 44, 1-5. II, 1, 192b28-29). Cuando estas causas o principios -no naturales en sentido estricto- actúan, sus efectos no son "por naturaleza" sino "por accidente", de tal modo que las propiedades adquiridas por su actuación no son "conforme a la naturaleza" (f. 44, 7-9. II, 1, 192b35). En este sentido, lo azaroso constituye un quiebre del orden de lo físico, una excepción no reglada cuyo estudio sobrepasa los limites de la ciencia tal como esta es comprendida por Aristóteles.

En el marco de las críticas que dirigió a las alternativas mecanicistas como respaldo a su teoría de las cuatro causas (f. 63, 1-8. II, 4, 195b31-35) ${ }^{8}$ -principalmente de la causa final-, entendidas como aquello que explica de modo suficiente el ser de lo natural (f. 53, 1-4. II, 194a21-26) ${ }^{9}$, el Filósofo definió lo azaroso como algo propio del ámbito de lo no-natural y de lo incognoscible

\footnotetext{
5 Valor, J., "Dialogando con la fisica de Aristóteles después de la Modernidad", en: González Recio, J. (coord.), Átomos, almas y estrellas: estudios sobre la ciencia griega, Madrid: Plaza y Valdés, 2007, p. 105.

6 "Signum autem est quod vanum est quoniam dicitur cum non fiat propter aliud illius causa ut ambulare depositionis causa et; si vero non fiat ambulanti frustra dicimus ambulasse et ambulatio vana est".

7 “...quod est natura principium alicuius est causa movendi et quiescendi in quo est primum per se et non secundum accidens".

8 "Dicitur autem et fortuna et casus causarum et multa et ese et fieri propter fortunam et propter casum: quodam igitur modo in his causis est fortuna et casus; et utrum eadem sit fortuna et casus aut altera, et omnio quid sit fortuna et casus, considerandum est".

9 "In antiques quidem igitur aspierent videbitur utique esse natura materiei; ex parva enim quadam parte Empedoclis et Democritus speciem et quod aliquid erat esse tetigerunt; si autem ars imitator naturam, eiusdem autem scientie est cognoscere speciem et materiam usque ad hoc (ut medici sanitatem et coleram et flegma, in quibus est sanitas; similiter autem et edificatoris est speciemque domus et materiam, quoniam lateres et ligna sunt; similiter autem et in aliis) et phisice erit cognoscere utrasque naturas".
} 
(Metafisica, f. 118, 21-22. VI,2, 1026b2-4) ${ }^{10}$. Aquello que se produce por azar no puede ser considerado como propiamente natural debido a que su acontecer lo revela como deficitario respecto de las propiedades constitutivas de lo físico. En este sentido, los procesos azarosos son irregulares, carecen de finalidad (Analiticos Posteriores, f. 87, 21. II, 11, 95a 8-9) y no poseen en sí mismos el principio de su movimiento ${ }^{11}$.

Este elevado grado de indeterminación imposibilita su aprehensión epistemológica por parte del ser humano, ya que la ausencia de regularidad, orientación teleológica o clara determinación formal impiden establecer con claridad las causas implicadas en su desarrollo (f. 69, 6-8. II, 5, 196b29) ${ }^{12}$.

Haciendo alusión, en este sentido, a las dificultades que la razón experimenta frente a la elusiva configuración ontológica de lo azaroso, Aristóteles definió el azar como una "causa divina y demoníaca e inmanifesta humano intellectul” (Fís. f. 67, 3-7. II, 4, 196b6) ${ }^{13}$. Dicho esto, es fácil intuir cómo, siendo consideradas por Aristóteles la razón y la explicación causal los métodos válidos para adquirir el conocimiento, lo azaroso y lo indeterminado quedan relegados al ámbito de lo irracional y lo oculto ${ }^{14}$.

Una vez constatado el reconocimiento de este ámbito de indeterminación cabe preguntarse por la posibilidad de que pueda ocurrir cualquier cosa al azar, es decir, que la fisica aristotélica pueda admitir la incidencia de cualquier fenómeno o la llegada al ser de cualquier tipo de entidad, o si más bien es necesario hablar de restricciones incluso en el ámbito de lo azaroso. Como mostraremos a continuación, la respuesta ofrecida por el Estagirita a esta cuestión es necesariamente negativa debido a la prevalencia de un conjunto de determinaciones inexorables.

\footnotetext{
10 "Signum autem: nulla enim scientia studiosa est de eo, non practica nec theorica". Por lo tanto, el carácter no necesario de lo accidental lo excluye como objeto de las ciencias demostrativas cuyos razonamientos deben partir de premisas necesarias. "Accidentium autem non per se, quo modo determinatum est per se quidem esse, non est scientia demostrativa" (Analiticos Posteriores. f. 18 22, f. 19,1. I, 75a 7-20).

$274{ }^{11}$ Una definición completa de estas caracteristicas de lo azaroso puede hallarse en la Retórica: "Sunt autem que a fortuna fiunt hec in quantum causa indeterminata et causa huius fiunt et quod neque Samper neque ut in pluribus nec ordinate (manifestum enim ex diffinitione fortune de hiis), natura vero quibuscumque causa est in ipsis et ordinata, si enim samper aut in pourbus simliter fiunt" (Rhetorica f. 44, 9-13. I 10, 1369a32-b5).

${ }_{12}$ "Per se quidem igitur causa finita est, secundum accidens autem infinita".

13 "Sunt autem quidam quibus videtur ese quidem causa fortuna, inmanifesta autem humano intellectui, sicut cum divinum quoddam sit et felicius".

${ }^{14}$ Rodríguez Donís, M., "Azar y finalidad en Aristóteles y Epicuro", en: Thémata. Revista de Filosofia, 18 (1997), pp. 55-64.
} 
El azar es entendido por Aristóteles como causa de entidades que pudiendo haber sido causadas por la naturaleza (f. 72, 3-7. II,5, 197a 22-24) ${ }^{15}$ lo han sido accidentalmente por otra cosa (f. 78, 1-7. II,6, 198a5-6) ${ }^{16}$. En este sentido, resulta evidente que este tipo de causas accidentales no pueden dar lugar a cualquier tipo de fenómeno, sino que, al manifestarse, sus posibles resultados se limitan a lo que igualmente podría ser producto de la naturaleza ${ }^{17}$. Las posibilidades son limitadas porque las potencias que cada cosa tiene según su naturaleza son también limitadas. Aristóteles insistió en esta idea acotando las posibles interacciones entre los cuerpos físicos al señalar que "no hay ninguna cosa que por su propia naturaleza pueda actuar de cualquier manera sobre cualquier otra al azar o experimentar cualquier efecto de cualquier cosa al azar y que cualquier cosa no puede llegar a ser cualquier cosa" (f. 23, 4-9. I 5, 188a32-36) ${ }^{18}$.

Establecida la estricta demarcación del campo de actuación del azar sostenida en la Física, quedan por analizar los casos en los cuales Aristóteles reconoció de forma abierta que en la naturaleza efectivamente pueden producirse fallos o desviaciones azarosas respecto del orden natural de los procesos, siendo ejemplo de ello la generación de lo monstruoso (f. 88, 2-f.89,1. II,8, 199b1-9) ${ }^{19}$.

La explicación de este tipo de fenómenos fue sustentada por el Estagirita en la noción de "fallo en la consecución del fin". Así, al igual que las cosas artificiales pueden ser hechas incorrectamente cuando el fin pretendido no se alcanza, en la naturaleza también pueden producirse errores consistentes en el defecto de algún principio en el proceso de generación. Concretamente, se trata de fallos derivados de la resistencia de la materia a ser informada adecuadamente 20 .

\footnotetext{
15 "Tamen autem in quibusdam deficiet utique aliquis, numquid igitur contingentia causa utique fiant fortune, ut sanitatis aut spiritus aut estus, sed non depilari; sunt autem alie aliis proximiores que sunt secundum accidens causarum".

16 "Quoniam autem sunt casus et fortuna cause quorum utique aut intellectus fiat causa aut natura, cum secundum accidens causa aliqua fiat horum ipsorum, nichil autem secundum accidens prius est eis que sunt per se, manifestum est quod neque secundum accidens causa prius est ea que est per se".

${ }_{17}$ Frede, D., "Accidental Causes in Aristotle", en: Synthese, 92, 1 (1992), pp. 39-41.

18 "Accipiendum igitur primum est quod omnium que sunt nichil neque facere aptum natum est neque pati contingens a contingenti, neque fit quodlibet ex quolibet, nisi aliquis accipiat secundum accidens".

19 "Si igitur sunt quedam secundum artem in quibus quod recte fit propter aliquid fit, in quibus autem peccatur alicuius quidem gratia esse argumentatur sed fallir simpliciter utique in phisicis et monstra sunt peccata illius quod propter aliquid est".

${ }^{20}$ Cencillo, L., Hyle: Origen, concepto y funciones de la materia en el Corpus Aristotelicum, Madrid: Consejo Superior de Investigaciones Científicas, 1958, p. 50.
} 
Por tanto, la explicación de los fallos azarosos es remitida por el Estagirita a su concepción de la materia entendida como pura potencialidad. El sustrato material que constituye, junto a la esencia o naturaleza, todos los mixtos sublunares, da lugar a la posibilidad de que las cosas sean de otro modo, o lo que es lo mismo, abre un espacio de discontinuidad en los procesos naturales respecto a la norma teleológica general pues, tal como señala Aristóteles en la Metafisica, lo que es por accidente (f. 128, 99-102. VI,2, 1026b30) tiene su causa en la materia (f. 129, 120-123. VI,2, 1027a10).

Sin embargo, lo fundamental de su reflexión radica en subrayar que cuando en virtud de la potencialidad de la materia surge un efecto inesperado, este resultado, si bien es fortuito, es en la misma medida connatural a las cosas naturales. Esta afirmación nos hace comprender cómo para Aristóteles, aunque los resultados monstruosos efectivamente se den, su configuración final también se halla radicalmente determinada por la forma y la materia (Fís. f.55, 5-6. II, 2, 194b9) ${ }^{21}$, de tal modo que no cabe cualquier tipo de aberración porque los posibles errores también se ven limitados al número finito de formas que la materia puede adquirir. En este sentido, la presencia del azar en la naturaleza aristotélica está significativamente coartada por las determinaciones cualitativas de las entidades respecto de las cuales se puede predicar la accidentalidad, y sensiblemente marcada por una visión negativa en la medida en que los procesos azarosos son difícilmente cognoscibles por la mente humana y sus resultados son habitualmente defectuosos e inferiores a los producidos por la naturaleza por medio de los cauces habituales.

No hay cabida a un azar absoluto en el cosmos teleológico de Aristóteles, ni causas más allá de las estrictamente determinadas en el libro II de la Física. Su visión de la fisica está marcada por la necesidad y la eterna regularidad en sus procesos, no siendo posible hablar de estados anteriores de mezcla, separación u origen temporal, y tampoco de una virtual disgregación de la actual configuración del mundo natural. Todo ha sido y será según la misma organización nomológica que la naturaleza muestra en el presente.

\section{El azar en la cosmología de Tomás de Aquino}

El problema de la presencia del azar en el mundo natural se vio aun más enfatizado en la Baja Edad Media cuando el pensamiento de Aristóteles entró en contacto con la visión cristiana del mundo según la cual el Ser necesario

21 "Amplius, eorum que sunt ad aliquid materia est, in alia enim specie alia materia iam est". 
había creado y determinado el orden de todo lo real22. En este sentido, el azar y lo casual parecen haber tenido, en el marco de la fisica medieval, un lugar todavía más reducido que en el pensamiento peripatético.

En el complejo y controvertido proceso de asimilación del pensamiento aristotélico que se produjo a lo largo del siglo XIII, la obra de Tomás de Aquino representó uno de los puntos centrales para la comprensión del desarrollo posterior de la ciencia física. Si bien el influjo del Filósofo sobre la obra política, ética y metafisica del Aquinate ha sido intensamente estudiado por la historiografia contemporánea, sus aportaciones en materia cosmológica no han sido objeto del mismo interés. Por ello, dedicaremos la segunda parte de este artículo a mostrar los puntos en los cuales Tomás de Aquino asimiló sin restricciones las posturas del Estagirita y aquellos en los cuales reformuló su pensamiento abriendo paso a nuevas vías de investigación vedadas en la cosmología de Aristóteles.

Partiendo de justificaciones distintas, ambos pensadores coincidieron en rechazar la idea de que el mundo pueda ser fruto del azar y que este tuviera un papel fundamental en la explicación de los fenómenos físicos. No obstante, al igual que en Aristóteles, la cuestión de la casualidad no fue totalmente rechazada por el Aquinate, sino analizada en profundidad en los fragmentos de su obra dedicados a cuestiones cosmológicas.

La definición más común de azar que hallamos en la obra del Doctor Angélico es aquella que lo vincula aristotélicamente con la noción de frustración en la consecución del fin. Según Tomás de Aquino, la actuación del azar puede ser verificada en aquellos casos en los cuales algo impide que el proceso natural se desarrolle alcanzando la finalidad intrínseca que dicta la esencia del ente implicado: "Por eso el azar, que en griego se dice automaton -esto es: frustrado por sí- sucede en aquellas cosas que existen por algo, y lo mismo lo frustrado o vano, porque es frustrado por sí y se frustra a sí mismo, según significa el nombre... cuando una piedra que cae golpea a alguien, no se dice que cayó para golpear, sino que cayó por lo que en sí es vano y frustrado, ya que por naturaleza no le es propio caer para eso" (Comentario a la Física, II, lect. 10, n. 157).

No obstante, esta definición excluye, para Tomás de Aquino, toda posibilidad de tomar como verdadera la propuesta de los presocráticos según la cual el universo ha tenido su origen en la actuación azarosa de la materia informe. Según el Aquinate, no es posible defender en materia física la teoría según la

${ }^{22}$ Alvira, R., "Casus et fortuna en Sto. Tomás de Aquino", en: Anuario Filosófico, X, 1 (1977), p. 32. 
cual mediante sucesivos procesos dinámicos de condensación y rarefacción una causa material haya dado lugar a la configuración actual del cosmos, porque si ello fuera cierto la interacción azarosa de partículas materiales preexistentes deberia ser tenida como fuente originaria y primer principio de la realidad ${ }^{23}$ : "Con esto se excluye la opinión de los antiguos naturalistas, que defendían que solo y únicamente la causa material era de la que se hacian todas las cosas, por enrarecimiento y densidad y así, era natural que estos dijesen que la distinción de las cosas que vemos en el universo no se verificó por una intención ordenadora, sino por el movimiento fortuito de la materia. Asimismo se excluye la opinión de Demócrito y Leucipo, que afirmaban la existencia de infinitos principios materiales, o sea, cuerpos indivisibles de la misma naturaleza, pero diferentes en sus figuras, orden y posición, de cuyo encuentro -que debía ser fortuito al negar la causa agente- decían que provenía la diversidad de las cosas, en razón de las diferencias dichas de átomos, esto es, de figura, de orden y de posición" (Suma contra gentiles, II, 39) ${ }^{24}$.

No obstante, el orden que presenta la naturaleza no puede responder, en opinión del Aquinate, a la mera casualidad, sino que su armonía se presenta como indicio racional de una organización inteligente. Las Escrituras, por su parte, establecían que en la creación no participó ningún tipo de materia, sino que el mundo fue creado de la nada por la acción divina. Por lo tanto, si la posibilidad de que el azar actúe como causa eficiente se vincula con la materia, la disposición de todas las cosas no puede ser fruto de este, ya que cuando los entes fueron creados y organizados no había ninguna materia preexistente y separada capaz de generar un ámbito de indeterminación gracias al cual poder justificar un origen azaroso de la naturaleza, sino que la materia -y con ello la posibilidad de que se desarrollen procesos azarosos- tuvo como origen la creación. De ello se sigue que hay una subordinación cronológica necesaria que obliga a que el azar sea algo propio del mundo ya creado y no un principio anterior al mismo: "Se demostró anteriormente que la primera producción

$278{ }^{23}$ Sakkopoulos, S.A. y E.G. Vitoratos, "Empirical Foundations of Atomism in Ancient Greek Philosophy", en: Science and Education, V, 3 (1996), pp. 293-303.

24 "Per haec autem excluditur opinio antiquorum Naturalium ponentium causam materialem solum et unam, ex qua omnia fiebant raritate et densitate. Hos enim necesse est dcere distinctionem rerum quas in universo videmus, non ex alicuius ordinante intentione provenisse, sed ex materiae fortuito motu. Similiter etiam excluditur opinio Democriti et Leucippi ponentium infinita principia materialia, silicet indivisibilia corpora eiusdem naturae sed differentia figuris, ordine et positione, ex quorum concursu - quem oportebat esse fortuitum, cum cauam agentem negarent- ponebant esse diversitatem in rebus, propter tres praedictas atomorum differentias, silicet figurae, ordinis et positionis; unde sequebatur distinctionem rerum esse fortuitam". 
de las cosas en el ser no proviene de la materia. Por donde el azar no puede tener cabida en ella. Ahora bien, es preciso que en la primera producción de las cosas hubiese distinción puesto que entre ellas se hallan muchas que no son engendradas mutuamente ni de una común, porque no coinciden en la materia. Luego no es posible que la distinción de las cosas sea azarosa" (Suma contra gentiles, II, 39) 25 .

En segundo lugar, el azar tampoco puede ser considerado, en opinión del Aquinate, como un principio activo capaz de influir sobre las cosas puramente inmateriales. Este tipo de entidades, carentes de contrariedad, se rigen exclusivamente por la necesidad derivada de su esencia. Por ello es posible constatar, según Tomás de Aquino, que en el mundo supralunar no hay espacio para el azar, ya que en esta región de la realidad todo acontece bajo el gobierno de las leyes que ordenan el movimiento de los astros y las esferas: "Lo azaroso no ocurre sino en lo que puede comportarse de distinto modo del que es, pues lo que ocurre necesariamente y siempre no decimos que sea azaroso. Ahora bien, se ha demostrado que hay algunas criaturas en cuya naturaleza no se da la posibilidad al no-ser, como son las sustancias inmateriales y sin contrariedad. Luego es imposible que sus sustancias sean por azar. Ahora bien, se distinguen mutuamente por su misma sustancia. Luego su distinción no es azarosa" (Suma contra gentiles, II, 39) 26.

En este sentido, si la distinción ontológica existente entre los diversos tipos de seres no puede ser remitida a la casualidad sino a la forma propia de cada ente, tampoco podrá considerarse el azar como principio rector de las cosas dotadas de esencia. En este caso, la naturaleza o forma de los entes es aquello que determina tanto su configuración cualitativa como el tipo y número de procesos que es capaz de ejercer y padecer. Pero si la forma rige la composición y comportamiento de las sustancias, como principio suficiente, no es necesario apelar a una causa indeterminada para explicarlas. El peso de la argumentación aristotélica en el posicionamiento del Aquinate en esta

\footnotetext{
25 "Ostensum est autem supra quod prima rerum productio in esse non est ex materia. In ea igitur casus locum habere non potest. Oportet autem quod prima rerum productio cum disctintione fuerti: cum multa inveniantur in rebus creatis quaae neque ex invicem generantur, neque ex aliquo uno communi, quia non conveniunt in materia. Non est igitur possibile quod rerum distinctio sit a casu".

26 "Casus enim non contingit nisi in possibilibus aliter se habere: quae enim sunt ex necessitate et semper, non dicimus esse a casu. Ostensum est autem supra quasdam res creatas esse in quarum natura non est possibilitas ad non esse: sicut sunt substantiae immateriales, et absque contrarietate. Substantias igitur eorm impossibile est esse a casu. Sunt autem per suam substantiam ab invicem distinctae. Earum igitur distinctio non est a casu".
} 
cuestión se muestra de forma clara e incontestable, tanto en la elección de las premisas fundamentales como en el modo en el que estas son empleadas en la argumentación: "Al darse solamente el azar en lo que puede comportarse de otro modo, y siendo el principio de esta posibilidad la materia y no la forma-que más bien determina la posibilidad de la materia a un modo de ser-, aquellas cosas cuya distinción depende de la forma no se distinguen azarosamente, sino solo quizá aquellas cuya distinción proviene de la materia. Ahora bien, la distinción de las especies proviene de la forma; la de los individuos de la misma especie proviene de la materia. Luego la distinción específica de las cosas no puede ser casual" (Suma contra gentiles, II, 39) ${ }^{27}$.

En tercer lugar, Tomás de Aquino acudió a la prioridad aristotélica de la causa per se frente a la causa per accidens, para restringir aun más la eficiencia del azar. Si hay una prioridad necesaria de las causas que son en sí mismas, de ningún modo se puede afirmar que la primera razón de la determinación del mundo físico pueda ser una o varias causas accidentales ${ }^{28}$. Aquello que está teleológicamente determinado antecede cronológicamente y precede ontológicamente a todo lo que se escapa a la determinación de los fines. La primacía de lo necesario y limitado es, aristotélicamente, incuestionable para el Doctor Angélico: "La causa por sí es anterior a la que es causa accidentalmente. Luego si las cosas posteriores proceden de una causa determinada por sí, no conviene decir que las anteriores proceden de una casa accidental indeterminada. Pero la distinción de las cosas precede naturalmente a los movimientos y operaciones de las cosas, pues a cosas determinadas y distintas corresponden movimientos y operaciones determinados. Ahora bien, los movimientos y operaciones de las cosas proceden de causas por sí y determinadas, puesto que hallamos que o siempre o en la mayoría de las veces proceden del mismo modo de sus causas" (Suma contra gentiles, II, 39) ${ }^{29}$.

\footnotetext{
27 "Cum casus sit tantum in possibilibus aliter se habere; principium autem huius possibilitatis est materia, non autem forma, quae magis determinat possibilitatem materiae ad unum; ea quoorum distinctio est a forma, non distinguuntur casu: sed forte ea quorum distinctio est a materia. Specierum autem distinctio est a forma: singularium autem eiusdem speciei a materia. Distinctio igitur rerum secundum speciem non potest esse a casu".

${ }^{28}$ Wallace, W.A., "Aquinas on the Temporal Relation Between Cause and Effect", en: Review of Metaphysics, 27 (1974), p. 570.

29 "Causa per se prior est ea quae est per accidens. Si igitur posteriora sint a caausa per se determinata, inconveniens est dicere priora esse a causa per accidens indeterminata. Distinctio autem rerum reaecedit naturaliter motus et operationes rerum: determinati enum motus et operationes sunt rerum determinatarum et disctinctarum. Motus autem et dissctintarum. Motus autem et operationes rerum sunt a causis per se et determinatis; cum inveniantur aut semper aut in pluribus ex suis causis eodem modo procedere".
} 
La cuarta razón aducida por el Aquinate se basa en la idea del orden del universo que es derivada argumentativamente de la infinita potencia e inteligencia divinas. Si Dios es un agente todopoderoso e inteligente, entonces ninguna parte de su obra puede ser remitida al azar porque precisamente lo azaroso es aquello que no está ordenado y que no presenta ningún rasgo intencional en su comportamiento ${ }^{30}$. Lo azaroso es caótico y, en general, deficiente, mientras que aquello que puede ser remitido a la obra del perfecto artesano tiene en sí mismo las marcas de la participación en la perfección absoluta (armonía, durabilidad y fecundidad): "Lo bueno y óptimo del universo consiste en el orden de las partes entre sí, el cual no puede darse sin distinción, pues por este orden se constituye el universo en su totalidad, que es lo óptimo de él. Luego el orden mismo de las partes del universo y la distinción de ellas es el fin de la producción del universo. En consecuencia, la distinción de las cosas no procede del azar" (Suma contra gentiles, II, 39) ${ }^{31}$.

En este punto, se hace evidente el hecho de que, para el Aquinate, el ámbito físico de operación del azar queda restringido al conjunto de los individuos sobre los cuales la fuerza de indeterminación de la materia puede influir en la configuración final de sus propiedades. Es en el plano de lo estrictamente corpóreo donde el azar se despliega y donde las conclusiones extraídas de su análisis tuvieron mayor relevancia para los intereses de la posterior ciencia moderna.

El concepto fundamental empleado por Tomás de Aquino para refutar definitivamente toda apelación al azar como causa explicativa suficiente del mundo o como principio fundamental de los procesos naturales fue el de providencia, con el cual se establece un orden necesario para la comprensión del mundo32: "Hubo quienes, como Demócrito y Epicuro, negaron en absoluto la providencia, porque suponian que el mundo había sido hecho por azar. Otros opinaron que solo los seres incorruptibles están sujetos a providencia y, en cuanto a los corruptibles, lo están las especies, que como tales son incorruptibles, pero no los individuos" (Suma teológica, 1, q.22, a.2) ${ }^{33}$.

\footnotetext{
${ }^{30}$ McGinn, B., "The Development of the Thought of Thomas Aquinas on the Reconciliation of Divine Providence and Contingent Action", en: The Thomist, 39 (1975), pp. 741-752.

31 "Sed bonum et optimum universi consistit in ordine partium eius ad invicem, qui sine distinctione esse non potest: per hunc enim orginem universum in sua totaliteta constituitur, quae est optimum ipsius. Ipse igitur ordo partium universi et disctinctio earum est finis productionis universi. Non est igitur distinctio rerum a casu".

${ }^{32}$ Blanchette, O., The Perfection of the Universe According to Aquinas: a Teleological Cosmology, Philadelphia: Pensylvania State University, 1992, pp. 29-30.

33 "Responde dicendum quod quidam totaliter providentiam negaverunt, sicut Democritus et Epicurei, ponentes mundum dactum esse casu. Quidam vero possuerunt incorruptibilia tantum
} 
Los individuos del mundo sublunar, por lo tanto, no han sido abandonados a merced del casu sino que Dios ejerce también en ellos su poder, conservando y determinando las cosas según un fin preestablecido: "es necesario decir que todos los seres están sujetos a la providencia divina, y no solo en conjunto, sino también en particular. La razón es porque, como todo agente obra por un fin, la ordenación de los efectos al fin se extiende hasta donde se extiende la causalidad del primer agente... Pero la causalidad de Dios, que es el primer agente, se extiende en absoluto a todos los seres, y no solo en cuanto a sus elementos específicos, sino también en cuanto a sus principios individuales, lo mismo si son corruptibles que si son incorruptibles" (Suma teológica, 1, q.22, a.2) ${ }^{34}$.

Esta defensa a ultranza de la providencia pone en jaque la posibilidad de hablar de azar en cualquiera de los sentidos ofrecidos por Aristóteles. Cabe preguntarse, entonces, cómo es posible llevar a cabo el acuerdo entre este gobierno absoluto de la divinidad y la aceptación de efectos derivados de la pura casualidad en la naturaleza cuya existencia, como se ha señalado más arriba, sí aceptó el Aquinate. ¿Hay algo capaz de sustraerse al efecto del dominio de Dios, o acaso esta determinación que parece contagiar la realidad es inevitable?

Tomás de Aquino rechazó toda posibilidad de hablar de un mundo regido por una necesidad absoluta e inmutable. La existencia de Dios no aboca la naturaleza a una mecánica infalible sino que es posible hallar resquicios gracias a los cuales la indeterminación puede expresar sus efectos.

El texto capital para comprender la concordia que el Aquinate intentó llevar a cabo entre los conceptos de azar y providencia se halla recogido en la cuestión 103 del primer libro de la Suma teológica. Los artículos séptimo y octavo se preguntan precisamente por la posibilidad de que algo pueda suceder fuera del gobierno divino. La respuesta del Aquinate consistió en una teoría mixta que ofrece un espacio para el fracaso en la consecución del fin atribuido siempre a las causas segundas, el cual, visto desde el punto de vista universal del Ser supremo, no constituye un acto que pueda considerarse como externo al orden divino.

providentiae subiacere; corruptibilia vero, non secundum individua, sed secundum species sic enim incorruptibilia sunt".

34 "Sed necesse est dicere omnia divinae providentiae subiacere, non in universali tantum, sed etiam in singulari. Quod sic patet. Cum enim omne agens agat propter finem, tantum se extendit ordinatio effectuum in finem, quantum se extendit causalitas primi agentis. Ex hoc enim contingit in operibus alicuius agentis aliquid provenire non ad finem ordinatum, quia effectus ille conseguitur ex aliqua alia causa, praeter intentionem agentis. Causalitas autem Dei, qui es primum agens, se extendit usque ad omnia entia, non solum quantum ad principia speciei, sed etiam quantum ad individualia principia, non solum inocrruptibilium sed etiam corruptibilium". 
La forma en la que es posible esta pequeña brecha por la cual se introduce una limitada indeterminación es expresada por Tomás de Aquino en una bella frase que nos hace entender cómo la necesidad sigue estando presente siempre como trasfondo de todas las operaciones naturales, incluso de aquellas que aparentemente puedan ser calificadas de azarosas: "Dios dispone todas las cosas con suavidad" (Suma teológica, 1, q. 103, a. 8).

En este sentido, Tomás remarca la posibilidad de que algo ocurra fuera del orden impuesto por una causa particular, pero ello no puede suceder en el caso de una causa universal. Para que el efecto propio de una causa particular no llegue a lograrse basta con que otra causa de la misma clase intervenga.

Sin embargo, dicha intervención -es decir, el abanico posible de interacciones causales- está contenida siempre dentro de lo determinado por el orden universal de las cosas. Al igual que ocurría en el caso de Aristóteles, pueden darse fenómenos azarosos, pero de ello no se sigue la afirmación de que cualquier cosa pueda pasar o existir. Habiendo una primera causa de todo el ser, es imposible que algo suceda en sentido absoluto en contra o fuera del orden divino.

Lo que se da es más bien, según Tomás, una mera impresión en la que "algo parece salirse en parte del orden de la providencia" cuando el observador atiende solo a la causa particular. Sin embargo, tanto el proceso como la causa discordante mediante la cual se produce el error o la imposibilidad de alcanzar el fin forman parte del orden natural establecido por Dios: "Cuando se dice que algunos efectos son contingentes, se entiende en orden a sus causas próximas, que pueden fallar en su obrar; no como si algo pudiera hacerse fuera totalmente del orden de la gobernación divina. El hecho mismo de que algo suceda fuera del orden de una causa próxima se debe a alguna otra causa sometida al gobierno divino" (Suma teológica, 1, q. 103, a. 8) ${ }^{35}$.

Si bien todo lo que tiene lugar en el mundo respeta el orden universal impuesto por la causa primera, ello no debe, de nuevo, llevarnos a considerar que Tomás de Aquino negó la existencia del azar y de sus consecuencias. En la cuestión diecinueve del libro primero de la Suma teológica, el Aquinate ofreció una explicación clara de por qué la divinidad desea la existencia de operaciones y procesos naturales que fracasen en su objetivo de lograr el fin propio.

\footnotetext{
35 "Dicendum quod dicuntur aliqui effectus contingentes, per comparationem ad proximas causas, quae in suis efectibus deficere possunt; non propter hoc quod aliquid fieri possit extra totum ordinem gubernationis divinae. Quia hoc ipsum quod aliquid conringit praeter ordinem causae proximae, est ex aliqua causa subiecta gubernationi divinae".
} 
La razón última remite a la necesaria o, mejor dicho, conveniente existencia de una jerarquía de las cosas que se ordenan desde lo más imperfecto hasta lo más perfecto, dando razón de ser y existencia al orden del universo: "Pues bien, Dios con objeto de que haya orden en los seres para la perfección del universo, quiere que unas cosas se produzcan necesaria y otras contingentemente, y para ello vinculó unos efectos a causas necesarias, que no pueden fallar y de las que forzosamente se siguen y otros a causas contingentes y defectibles" (Suma teológica, 1, q. 19, a. 8) ${ }^{36}$.

Tal y como señala Alvira ${ }^{37}$, el Aquinate transformó de esta forma la noción de azar, que en Aristóteles era sinónimo de error y fracaso, en un concepto positivo que expresa el bien del que está dotado el universo creado por Dios. De este modo, la actuación aleatoria de las causas segundas en algunos casos concretos no constituye una merma en el orden del universo, sino que su misma existencia lo garantiza. La providencia divina solo tiene sentido en un mundo en el cual no todos los procesos se desarrollan de forma necesaria, sino que hay cabida para la contingencia y la indeterminación: "Se dice que hay fortuna o azar en aquellas cosas que suceden pocas veces, pues de no haber cosas así todo acontecería por necesidad, porque las cosas contingentes solo se suelen diferenciar de las necesarias en que aquéllas a veces pueden fallar. Además, si todo acontece necesariamente, se destruiría el concepto de providencia, según consta. Por lo tanto, el suponer que no se da nada fortuito o casual es contra el concepto de providencia divina"38.

Los agentes secundarios que animan el orden natural pueden fallar en sus objetivos con vistas a los fines propios. Pero si esto no sucediera, según Tomás, no habría ni providencia ni verdadera perfección del universo.

Sin embargo, si bien desde un punto de vista universal la existencia de efectos azarosos es considerada como necesaria para hablar de la perfección de la obra total, Tomás de Aquino reconoció que dichos efectos pueden considerarse como negativos para los entes que los padecen.

\footnotetext{
36 "Vult autem quaedam fieri Deus necessario, et quaedam contingenter, ut sit ordo in rebus, ad complementum universi. Et ideo quibusdam effectibus aptavit casuas necessarias, quae deficere non possunt, ex quibus effectus de cecessitate proveniunt: quibusdam autem aptavit causas contingentes defectibiles, ex quibus effectus contingenter eveniunt".

37 Alvira, R., Casus et fortuna en Sto. Tomás de Aquino, p. 64.

38 "In his enim quae in minori parte accidunt, dicitur esse fotuna et casus. Si autem non provenirent aliqua ut in minori parte, omnia ex necessitate acciderent: nam ea quae sunt contingenia ut in pluribus, in hoco solo a necessariis differunt, quod possunt in minori parte deficere. Esset autem contra rationem providentiae divinae si omnia ex necessitate contingeret, ut ostensum est" (C.G. III, c.74).
} 
Para diferenciar las cosas que son producto de las operaciones naturales exitosas de las que surgen como consecuencia de un fallo en la concatenación causal, el Aquinate empleó el término contra natura. Los seres que pertenecen a esta clase se identifican por mostrar algún defecto de la virtud operativa, como es el caso, por ejemplo, de los individuos que nacen cojos o ciegos. Ni la ceguera ni la cojera son, según el Aquinate, fines intentados por la naturaleza, sino que esto sucede fuera de la intención de la naturaleza y por defecto de los principios naturales ${ }^{39}$.

En la tercera Cuestión disputada sobre el poder de Dios, Tomás de Aquino atribuyó un sorprendente origen al conjunto de los defectos que pueden mostrar los entes físicos. Apartándose en este punto de la doctrina aristotélica, el Aquinate señaló que la razón última de los fallos no está ni en la resistencia de la materia ni mucho menos en la voluntad divina, sino en una especie de impronta que la nada ha dejado en este tipo de entidades: "Si existe alguna imperfección en las criaturas, no es necesario que proceda de Dios o de la materia, sino que se dará en cuanto que la criatura procede de la nada" (Cuestiones disputadas sobre el poder de Dios, 3, a1, ra14), es decir, el mal que para Tomás de Aquino se encuentra en muy pocas cosas y se debe a algún tipo de impedimento que le sobreviene a la causa agente $(Q D P 3$, a6, ra5) no es naturaleza alguna pero tampoco es pura negación sino una privación ${ }^{40}$. Esta privación heredada de la nada originaria por los seres capaces de experimentar la contradicción es caracterizada aristotélicamente como una negación que inhiere en algún sujeto, ya que la privación es una negación en la sustancia (QDP 3, a6, ra7). La incidencia de este tipo de desviaciones suele dar lugar a resultados negativos desde el punto de vista de los individuos afectados pero no desde el punto del fin universal de lo creado: "El orden impuesto por Dios a las cosas se desenvuelve según el curso normal de las cosas, mas no en todo momento y sin excepción, pues muchas de las causas naturales producen frecuentemente sus efectos del mismo modo, pero no siempre, pues a veces, aunque en contados casos, sucede lo contrario... por ejemplo cuando la naturaleza engendra en el hombre un sexto dedo. Sin embargo, por eso no falla o cambia el orden de la providencia, pues es cosa sometida a la divina providencia

\footnotetext{
39 "Dicit tamen quorum opus est, propter ea quae sunt contra naturam, sicut sunt monstra; quorum non est aliquod opus inquantum huiusmodi, sed patiuntur defectum operativae virtutis, ut patet in his qui nascuntur claudi vel caeci; non enim claudicatio est finis intentus a natura, propter quem faciat nasci animal claudum, sed hoc accidit praeter intentionem naturae ex defectu naturalium principiorum" (In De Coelo II lect. 4 n. 334).

40 Davies, B., Thomas Aquinas on God and Evil, Nueva York: Oxford University Press, 2011, p. 65.
} 
que el orden natural, establecido en atención a lo que sucede frecuentemente, falle alguna vez" (Suma contra gentiles, III, 99) ${ }^{41}$.

La confluencia de estos dos poderosos sistemas de pensamiento generó, a pesar del intenso proceso de condenas al que dio lugar en el siglo XIII, un nuevo marco en el cual podían plantearse como objetos de estudio propios de la fisica cuestiones que no preocuparon ni a los pensadores medievales anteriores a la llegada del corpus ni a la propia física de Aristóteles. Lo anómalo, lo indeterminado, lo infrecuente, y todo aquello que parecía romper las reglas de juego de la cosmología del Estagirita, comenzaron a centrar el interés de los pensadores al ser dotados, desde el plano cristiano, de un sentido positivo y explicativo para la comprensión de la realidad.

Aristóteles dotó a la Baja Edad Media de las herramientas lógicas y teóricas necesarias para volver a considerar la naturaleza como un campo de estudio valioso e independiente de la teología ${ }^{42}$, mientras que muchas de las premisas cristianas contra las cuales chocaban las tesis del Filósofo sirvieron para romper algunas de sus posiciones más rígidas y abrir nuevos campos de estudio. La cuestión del azar, que en este periodo temprano muestra solo algunas de sus decisivas implicaciones para la Modernidad en una discusión eminentemente teórica, habría de convertirse en una de las palancas fundamentales para la consideración del mundo como un mecanismo mucho más complejo y diverso del que había imaginado Aristóteles. El descubrimiento de un nuevo continente -con especies de plantas y animales desconocidos-o de nuevos cuerpos celestes -con una configuración muy diferente a la establecida por el Filósofo- acabó por quebrar las bases de su sistema físico, pero mucho antes de que estas evidencias pudieran ser tomadas en cuenta por los pensadores, la Baja Edad Media comenzó a incubar las premisas para la llegada de un nuevo mundo para la física.

Recibido: $28 / 07 / 2017$

Aceptado: 27/05/2018

\footnotetext{
41 "Ordo enim inditus rebus a Deo, secundum id est quod in rebus frequenter accidere solet, non autem ubique secundum id quod est semper; multae enim naturalium causarum effectus suos producunt eodem modo ut frequenter, non autem ut semper; nam quandoque licet ut in paucioribus... sicut cum natura in homine generat digitum sextum. Non autem propter hoc deficit aut mutatur providentiae ordo: nam et hoc ipsum quod naturalis ordo, institutus secundum ea quae sunt frequenter quandoque, deficiat, providentiae subest divinae".

42 Eamon, W., Science and the Secrets of Nature: Books of Secrets in Medieval and Early Modern Culture, Princeton: Princeton University Press, 1994, pp. 80-85.
} 


\section{Bibliografia}

Alvira, R., "Casus et fortuna en Sto. Tomás de Aquino", en: Anuario Filosófico, 10, 1 (1977), pp. 27-69.

Aristóteles, Aristoteles latinus. Rhetorica, translatio Guillelmi de Moerbeka edidit Bernhardus Schneider, Leiden: Brill, 1978.

Aristoteles latinus. VII. 1.1-2 Physica / edition curandae praesidet G.Verbeke, Leiden: Brill, 1990.

Aristoteles latinus. Vol. XXV 3, 2, Metaphysica lib. I-XIV. 2, Editio textus / edition curandae praesidet G.Verbeke; recensio et translatio Guillelmi de Moerbeka; edidit Gudrun Vuillemin-Diem, Leiden: Brill, 1995.

Blanchette, O., The Perfection of the Universe According to Aquinas: a Teleological Cosmology, Philadelphia: Pensylvania State University, 1992.

Cencillo, L., Hyle: Origen, concepto y funciones de la materia en el Corpus Aristotelicum, Madrid: Consejo Superior de Investigaciones Científicas, 1958.

Cunningham, H-P., Négation de la négation, A propos de "hazard" et de "nécésité", Quebec: Lava, 1972.

Davies, B., Thomas Aquinas on God and Evil, Nueva York: Oxford University Press, 2011. https://doi.org/10.1093/acprof:oso/9780199790890.003.0010

Eamon, W., Science and the Secrets of Nature: Books of Secrets in Medieval and Early Modern Culture, Princeton: Princeton University Press, 1994.

Frede, D., “Accidental Causes in Aristotle”, en: Synthese 92, 1 (1992), pp. 39-62. https:// doi.org/10.1007/bf00413741

McGinn, B., "The Development of the Thought of Thomas Aquinas on the Reconciliation of Divine Providence and Contingent Action", en: The Thomist, 39 (1975), pp.741752. https://doi.org/10.1353/tho.1975.0073

Rodríguez Donís, M., “Azar y finalidad en Aristóteles y Epicuro", en: Thémata. Revista de Filosofia, 18 (1997), pp.55-64.

Sakkopoulos, S.A. y Vitoratos, E.G., "Empirical Foundations of Atomism in Ancient Greek Philosophy”, en: Science and Education, 5 (3) (1996), pp. 293-303. https://doi. org/10.1007/bf00414318

Tomás de Aquino, Suma contra gentiles, I. Libros $1^{\circ}$ y $2^{\circ}$, Dios, su existencia, su naturaleza; La creación y las criaturas, Madrid: Biblioteca de Autores Cristianos, 2007.

- Suma teológica, I. Tratado de Dios es uno, Madrid: Biblioteca de Autores Cristianos, 2010.

Cuestiones disputadas sobre la potencia de Dios, cuestión 3: la creación, Pamplona: Ediciones de la Universidad de Navarra, 2011.

Valor, J., "Dialogando con la fisica de Aristóteles después de la Modernidad”, en: González Recio, J. (coord.), Átomos, almas y estrellas: estudios sobre la ciencia griega, Madrid: Plaza y Valdés Editores, 2007, pp. 87-122.

Wallace, W.A., "Aquinas on the Temporal Relation between Cause and Effect", en: Review of Metaphysics, 27 (1974), pp. 569-584. 